\title{
Description d'Oncbocerca dewittei n. sp. (Filarioidea) chez Sus scrofa en Malaisie (1)
}

\author{
par Odile BAIN *, C. P. RAMACHANDRAN **, F. PETTER *** \\ et J. W. MAK **** \\ * Laboratoire de Zoologie (Vers) associé au C.N.R.S., \\ Muséum national d'Histoire naturelle, 43, rue Cuvier, F 75231 Paris Cedex 05. \\ ** School of Biological Sciences, University Sains, Malaysia, Penang. \\ *** Laboratoire de Zoologie (Mammifères et Oiseaux), \\ Muséum national d'Histoire naturelle, 55, rue de Buffon, F 75005 Paris. \\ *a*k* Institute for Medical Research, Filariasis division, Jalan Pahang, \\ Kuala Lumpur, Malaisie.
}

\section{Résumé.}

Onchocerca dewittei n.sp. est récoltée chez le Sanglier au niveau des métatarses (tendons et conjonctif sous-cutané) ; elle est distincte par la cuticule de la $q$, à côtes rectilignes se chevauchant dans les champs latéraux et la microfilaire relativement épaisse (228-247 $\mu$ de long et 6-7 $\mu$ de large). Cette Onchocerque de Suidé présente certains caractères primitifs (côtes rectilignes, persistance de dix paires de papilles caudales chez le $\left.\delta^{\star}\right)$, mais est dans l'ensemble nettement plus évoluée qu'O. raillieti Bain, Müller et coll., 1976, parasite d'Equidés.

\section{Summary:}

Description of Onchocerca deivittei n. sp. (Filarioidea), parasite of Sus scrofa, in Malaysia.

Onchocerca dewittei n. sp. was collected from a wild Boar at the metatarse level (tendons and subcutaneous connective tissue); it can be differentiated from other species Santé.

(1) Ce travail a pu être effectué grâce à une subvention de l'Organisation Mondiale de la

Reçu le 15 février 1977. 
by the female cuticle showing straight ridges which overlap in the lateral fields, and by its relatively thick microfilaria (length 228-247 $\mu$ and width 6-7 $\mu$ ). This suidean Onchocerca displays some primitive characters such as straight ridges and persistency of ten pairs of caudal papillae in the male; but as a whole this species is undoubtedly more highly evolved than O. raillieti Bain, Müller and coll., 1976, a parasite of Equidae.

\section{Introduction}

En 1953, aux Indes (Madras), Ramanujachari et Alwar signalent la présence dans l'aorte du Porc domestique d'une Onchocerque, Onchocerca sp. ; la microfilaire n'est pas connue.

En 1967, en Malaisie, Ramachandran et Tan décrivent une microfilaire de type Onchocerca, située dans la peau dorsale d'un Porc; elle est longue de 300-340 $\mu$ et large de 8,5-10 $\mu$ (mensurations après coloration au Giemsa).

En octobre 1976, nous récoltons chez un Sanglier en Malaisie une Onchocerque située au niveau des pattes ; la microfilaire est longue de $200 \mu$ et large de $6 \mu$ (mensurations effectuées aussi après coloration au Giemsa).

Il y a donc certainement deux et peut-être trois espèces d'Onchocerques chez Sus scrofa, ce qui n'est pas surprenant, puisque l'on connaît, par exemple, trois espèces chez le Cheval, sept espèces chez le Bœuf et quatre chez le Cerf d'Europe.

Nous décrivons ci-dessous l'Onchocerque que nous avons récoltée dans les pattes du Sanglier de Malaisie.

\section{Matériel :}

Les cochons que nous avons examinés sont des sangliers sauvages vivant en pleine forêt, abattus pour être vendus sur le marché de Kuala Lumpur. Nous avons disséqué un Sanglier entier (négatif) et neuf pattes provenant de quatre animaux différents ; les microfilaires ont été recherchées dans des fragments de peau dorsale de six sangliers.

Hôte: Sus scrofa jubatus, Miller.

Provenance: réserve de Krau, Etat de Pahang, Malaisie.

Localisation: les adultes ont été trouvés dans un pied; ils s'étendent du tarse aux sabots et sont situés, soit dans l'épaisseur d'un tendon ( $q 1$ et $\delta 1$ ), soit entre un des tendons et sa gaine, entièrement libre ( $q 2$ ), soit dans le conjonctif ( $q 3$ et $\delta$ 2) ; la 3 était située dans un tube réactionnel, plus ou moins enroulé sur lui-même et dont les boucles étaient soudées par du conjonctif, l'ensemble formant une sorte de kyste

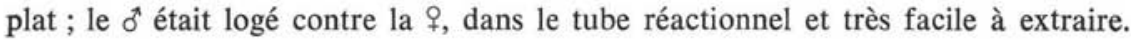

Les microfilaires dermiques ont été trouvées à deux reprises; dans un seul cas, les microfilaires étaient très nombreuses et bien mobiles, abondamment distribuées de la base de la queue à la nuque. 
Spécimens : 3 et 2 б.

Holotype $q 3$ entière et allotype $\delta 2$; $q 2$ incomplète (pas de région caudale) mais avec des microfilaires dispersées le long des utérus; +1 incomplète (pas de tête) et associée au mâle 1 (lot $100 \mathrm{JE}$, conservé au Muséum national d'histoire naturelle).

\section{Description}

Femelle (fig. 1).

Le corps présente quelques ondulations très lâches, la région antérieure est longuement effilée, la région postérieure est faiblement mais plus brusquement amincie, la queue est recourbée dorsalement.

Au niveau de l'anneau nerveux, il existe un renflement cervical, plus marqué sur la face ventrale, là où se trouve le pore excréteur. La cuticule est mince et finement striée dans la région cervicale, dans le reste du corps elle est épaisse, divisée en deux couches inégales, la couche interne est la plus épaisse (aucune strie n'a pu être mise en évidence) ; la couche externe est ornée de côtes bien saillantes rectilignes, régulièrement espacées, qui s'interrompent dans les plans latéraux, de telle sorte que les demi-anneaux d'une face du corps s'intercalent entre les demi-anneaux de la face opposée; ces demi-anneaux se chevauchent au niveau des champs latéraux. Les côtes restent très saillantes dans la région postérieure mais elles sont plus serrées.

La tête est arrondie, la position des papilles est irrégulière et présente quelques variations : chez la $q$ holotype $(q 3)$, les papilles labiales externes en vue latérale sont à peu près sur le même axe vertical que les papilles céphaliques, ou même un peu plus écartées des amphides que ces dernières; chez la $q 2$, la vue apicale montre que trois des papilles labiales externes sont moins écartées du plan latéral que les papilles céphaliques et que la quatrième est au contraire nettement plus éloignée de ce plan. Les amphides ont la forme de poche, comme chez toutes les Onchocerques. La bouche est ronde; le canal qui la relie à l'œsophage a une section triangulaire ; il est limité à sa base par une lame cuticulaire. L'œsophage est long, très mince et sans différenciation musculo-glandulaire. La vulve est située à la fin du tiers antérieur de l'œsophage; le corps est légèrement épaissi sur la face ventrale, en arrière de la vulve ; vagin et ovéjecteur sont fins et dirigés vers l'arrière.

La queue présente une légère torsion sur son axe vers l'extrémité (les phasmides se présentent en vue latérale quand l'anus est en position ventrale) ; autour de l'anus, les côtes s'interrompent et sont remplacées par des rides irrégulières; l'extrémité caudale est conique, obtuse, ornée par un appendice ventral terminal, en forme de goutte et par les deux phasmides épaisses, un peu saillantes et de forme simple.

\section{Dimensions.}

@ holotype ( $(3)$ : corps long de $30,7 \mathrm{~cm}$ et large de $340 \mu$ au milieu du corps ; anneau nerveux et vulve respectivement à 240 et $570 \mu$ de l'extrémité céphalique; œesophage long 


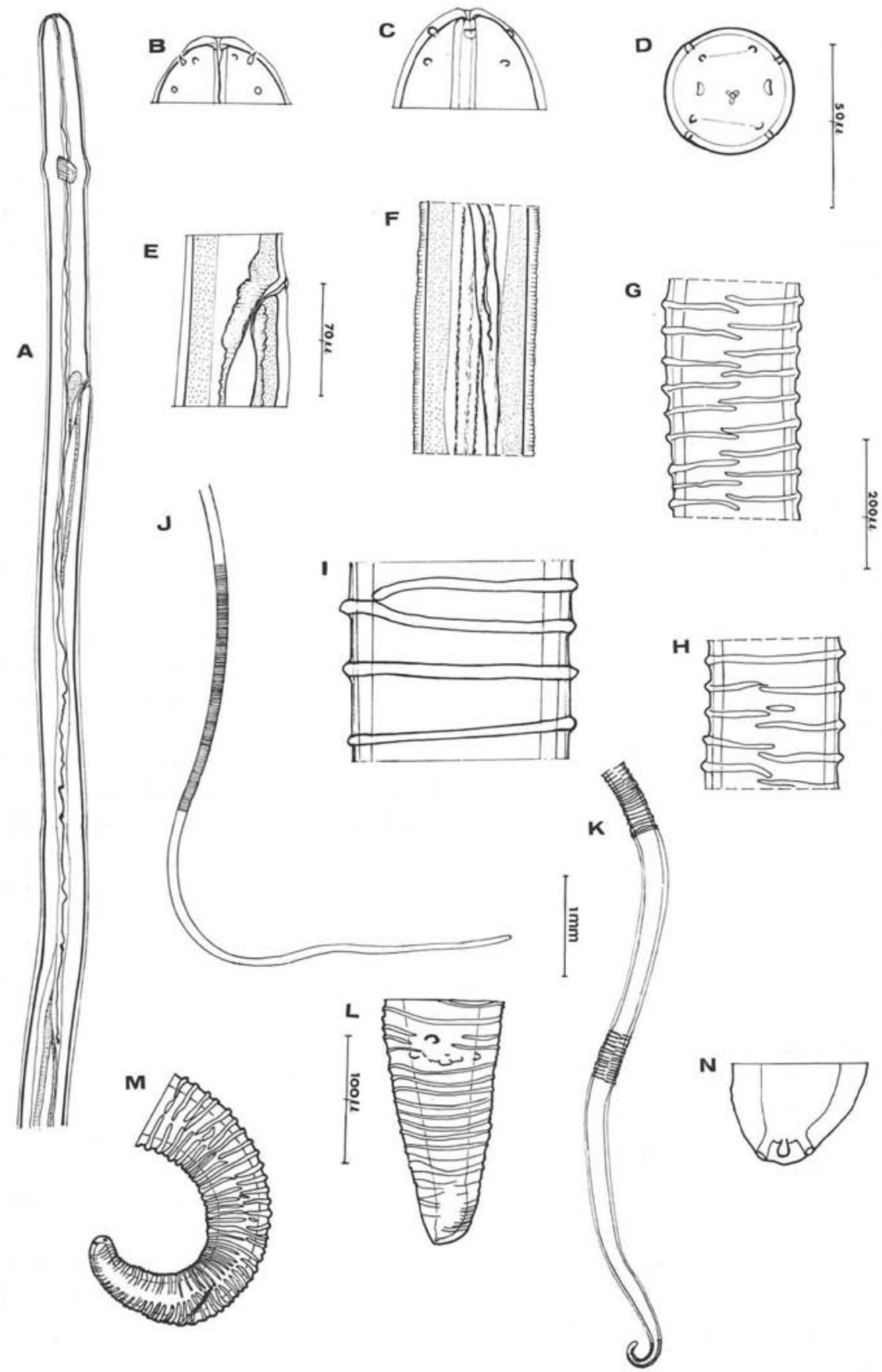

FIG. 1. - Onchocerca dewittei n. sp. femelle; A : région antérieure, vue latérale; B, C et D : tête en vues médiane, latérale et apicale; E : région vulvaire, vue latérale ; F : jonction œsophage-intestin; $\mathrm{G}$ et $\mathrm{H}$ : ornementation cuticulaire, vue latérale; $\mathrm{I}$ : idem, diamètre maximum, vue médiane; $\mathbf{J}$ et $\mathrm{K}$ : extrémités antérieure et postérieure du ver; $\mathbf{L}$ : région caudale, vue médiane au niveau de l'anus; $\mathrm{M}$ : queue, vue latérale au niveau de l'anus; $\mathrm{N}$ : extrémité caudale (vue ventrale). (A, G, I, H et M: éch. $200 \mu ; \mathrm{B}, \mathrm{C}, \mathrm{D}$ et $\mathrm{N}$ : éch. $50 \mu ; \mathrm{E}$ et $\mathrm{F}$ : éch. $70 \mu ; \mathrm{J}$ et $\mathrm{K}$ : éch. $1 \mathrm{~mm} ; \mathrm{L}$ : éch. $100 \mu$ ). 
de $1600 \mu$ sur $80 \mu$ de large ; cuticule épaisse de $40 \mu$; côtes espacées de 48-65 $\mu$, larges de $15-20 \mu$ et faisant une saillie de 8-12 $\mu$; queue longue de $190 \mu$.

2 : anneau nerveux et vulve à 240 et $700 \mu$ de l'extrémité antérieure ; œsophage long de $1400 \mu$; sur un fragment large de $220 \mu$, côtes espacées de $20 \mu$ et larges de $15 \mu$; portion impaire de l'ovéjecteur longue de $7200 \mu$.

Queue de la $\subsetneq 1$ longue de $162 \mu$.

Mâle (fig. 2).

L'état de conservation des $2 \sigma^{\circ}$ est insuffisant pour permettre une étude morphologique de la région antérieure. Seul le renflement cervical est visible. Par contre, les régions caudales sont restées en bon état. La queue porte deux ailes latérales; il existe une papille impaire précloacale et dix paires de papilles : deux paires nettement précloacales, éloignées l'une de l'autre (sur le $\delta^{\star} 1$, une papille de la première paire a disparu), puis quatre paires groupées au niveau ou en arrière du cloaque (trois paires sont latérales et une est ventrale) ; enfin quatre paires étagées le long de la moitié distale de la queue. La dixième paire de papilles est située sur une protubérance terminale; à la base de la $9^{\circ}$ paire se trouvent les phasmides canaliculaires. La septième paire de papilles est très réduite; la quatrième paire et la paire ventrale postcloacale ( $\mathrm{n}^{\circ} 5$ ou 6 ?) sont également moins développées que les autres papilles.

Le spicule gauche se termine par une pointe conique peu effilée. Le spicule droit porte, comme chez les autres Onchocerques, deux ailes latéro-ventrales; il présente une encoche dorsale; la spatule terminale est trapue avec un talon bien marqué mais non récurrent.

\section{Dimensions :}

$\delta^{*}$ allotype $\left(\sigma^{2} 2\right)$ : corps long de $4,3 \mathrm{~cm}$ et large de $90 \mu$; queue longue de $82 \mu$; spicules gauche et droit longs respectivement de $215 \mu$ (manche long de $105 \mu$ ) et $85 \mu$.

$\delta^{2} 1$ : corps long de $4 \mathrm{~cm}$ et large de $100 \mu$; queue longue de $80 \mu$; spicules gauche et droit longs respectivement de $250 \mu$ et $82 \mu$.

\section{Microfilaires (fig. 2).}

Extraites de l'utérus de la $q 2$ fixée à l'alcool : corps long de 230 et $238 \mu$, large de 6,5-7 $\mu$; cuticule épaisse ; tête arrondie ou un peu en biseau suivant l'orientation ; crochet assez développé; espace céphalique long de $5 \mu$; anneau nerveux à $55 \mu$ de l'apex ; extrémité caudale anucléée courte $(12 \mu)$ à pointe mousse.

Récoltées dans le derme, en coloration vitale : corps long de $228 \mu, 240 \mu$ et $247 \mu$ sur $6,5 \mu$ de large. Pour une microfilaire longue de $247 \mu$, noyau excréteur et R1 respectivement à 72 et $173 \mu$.

Sur une préparation fixée et colorée au glychemalum de Mayer, corps long de $200 \mu$ et large de $6 \mu$. 
A

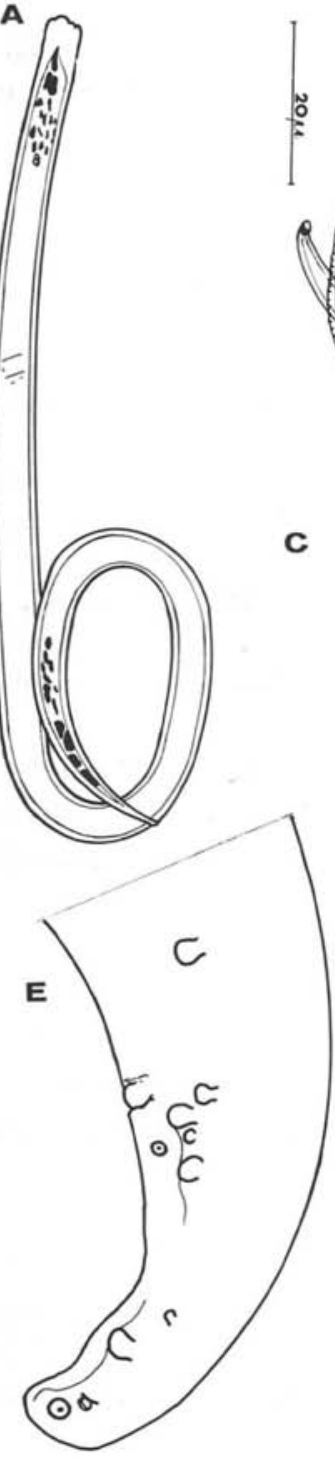

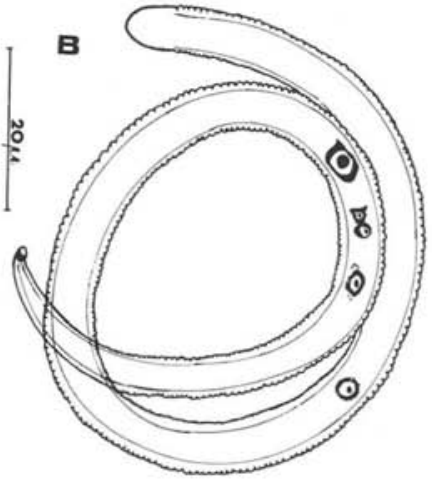

í

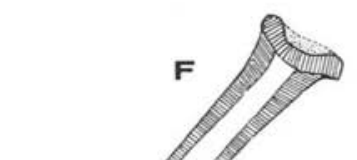

D

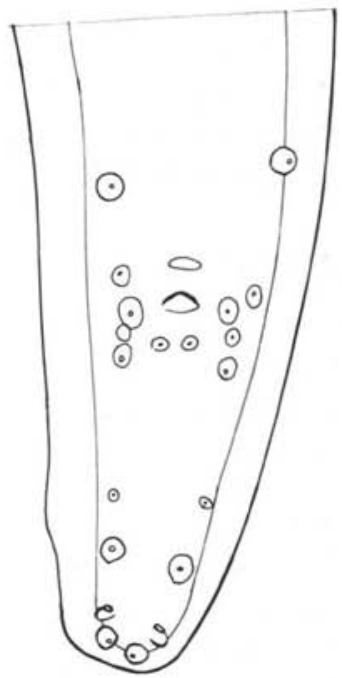

$\mathbf{G}$

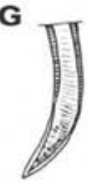

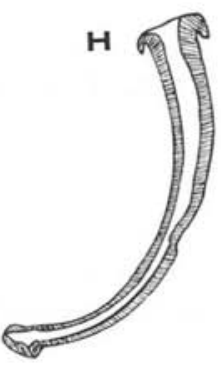

I

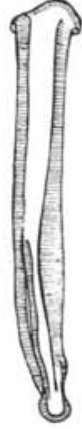

Fic. 2. - $O$. dewittei n. sp. A et $\mathrm{B}$ : microfilaire, - fixée à l'alcool et récoltée dans l'utérus - , dermique et colorée vitalement (la position des noyaux $R_{2}, R_{3}$ et $R_{4}$ est incertaine); C : tête de la microfilaire, crochet vu de profil; $\mathrm{D}$ à I : mâle; D et E : les queues des $2 \sigma^{t}$ en vues ventrale et latérale; $F$ : spicule gauche, vue latérale; $G$ : idem, extrémité distale ; H et I : spicule droit, vues latérale et ventrale. (A, D, E, F, G, H et I : éch. $50 \mu$; B et $\mathrm{C}$ : éch. $20 \mu$ ). 


\section{Discussion}

Le genre Onchocerca comprend 21 espèces suffisamment connues pour permettre une comparaison avec notre matériel.

O. fasciata Railliet et Henry, 1910, parasite de Camélidés, a un corps plus large que nos spécimens et des côtes très ondulées.

O. reticulata Diesing, 1841, O. cervicalis Railliet et Henry, 1910, O. raillieti Bain, Müller et coll., 1976, parasites d'Equidés et $O$. flexuosa (Wedl, 1856), parasite de Cervidés, s'opposent d'emblée à nos spécimens par leur œsophage développé (longueur supérieure à $2 \mathrm{~mm}$ chez la $q$; portion glandulaire bien distincte) et la vulve bien antérieure (distance à la tête égale ou inférieure au double de la distance tête-anneau nerveux).

O. armillata Railliet et Henry, 1909, parasite de Bovidés, est également bien distinct de notre matériel par l'œsophage très développé.

Parmi les Onchocerques qui ont un œsophage peu développé (longueur inférieure à $2 \mathrm{~mm}$ chez la $q$; portion glandulaire généralement indistincte), 12 espèces s'opposent à nos spécimens par l'absence de renflement cervical, la morphologie différente de la microfilaire, et certains autres caractères qui varient selon les espèces :

- O. bohmi (Supperer, 1953) chez les Equidés, O. ochengi Bwangamoi, 1969 et O. lienalis (Stiles, 1890) chez les Bovins n'ont pas de côtes transversales cuticulaires chez la ?.

- O. volvulus (Leuckart, 1893) chez l'Homme et $O$. dukei, Bain et coll., 1974, parasite de Bovins en Afrique, ont des côtes rectilignes mais interrompues dans les champs latéraux et un nombre plus réduit de papilles caudales.

- O. gibsoni Cleland et Johnston, 1910 et l'espèce proche O indica Sweet, 1915, parasites sous-cutanés de Bovidés, ont des $q$ plus larges, les dimensions des queues des $\delta$ et des spicules plus petits.

- O. cebei Galliard, 1937, en Asie, et $O$. sweetae Spratt et Moorhouse, 1971, en Australie, toutes deux parasites intradermiques des Bovidés, ont un spicule gauche particulièrement grand.

- O. tubingensis Bain et Schulz-Key, 1974, à œsophage nettement divisé bien que très court, $O$. jakutensis (Gubanov, 1964), très proche de l'espèce précédente et O. garmsi Bain et Schulz-Key, 1976, toutes parasites de Cervidés en Europe, ont des côtes ondulées et interrompues sur toute la largeur des champs latéraux.

Parmi les trois Onchocerques qui ont un renflement cervical, O. cervipedis Wehr et Dikmans, 1935, parasite de Cervidés nord-américains, est bien distinct de nos spécimens par l'absence de côtes cuticulaires chez la $\$$; $O$. tarsicola Bain et Schulz-Key, 1974, parasite du Cerf d'Europe et $O$. gutturosa Neumann, 1910, chez les Bovins, dont les femelles ont une cuticule ornée de côtes, sont les deux espèces qui se rapprochent le plus de l'Onchocerque du Sanglier. Toutefois, elles en diffèrent par les côtes ondulées, interrompues dans les champs latéraux et par d'autres caractères : 
- chez O. tarsicola, la microfilaire est plus grande $(400-430 \mu$ sur $12 \mu$ de large au lieu de 200-247 $\mu$ sur $6,5 \mu$ ), la queue du $\sigma^{*}$ et le spicule droit sont plus longs, le spicule gauche est plus court, les papilles caudales sont moins nombreuses et leur disposition est irrégulière ;

- chez $O$. gutturosa, les papilles labiales externes sont particulièrement éloignées de la bouche (en vue apicale, elles apparaissent sur le même cercle que les papilles céphaliques in Bain, 1975), la microfilaire est plus grêle $(5 \mu$ de large au lieu de $6,5 \mu)$, la queue du $\sigma^{t}$ et le spicule gauche sont plus longs, les papilles caudales sont moins nombreuses $\left(10^{\circ}\right.$ paire absente, $9^{\circ}$ paire réduite à deux pointes cuticulaires) et leur disposition est plus irrégulière.

Nos spécimens constituent donc une espèce nouvelle, $O$. dewittei n. sp., que nous dédions au $\mathrm{D}^{r}$ Dewitte, Directeur de l'Institute for Medical Research, à Kuala Lumpur.

Cette espèce est caractérisée par la cuticule de la $q$ à côtes rectilignes qui se chevauchent sur les champs latéraux, la microfilaire particulièrement large, les dix paires de papilles caudales présentes dont six rassemblées près du cloaque.

\section{Conclusion}

Assez fréquemment dans l'évolution des Nématodes, les parasites de Suidés ont des caractères primitifs par rapport aux parasites d'Artiodactyles. Les côtes non ondulées chez la + , la persistance du nombre primitif des dix paires de papilles caudales chez le $\delta^{*}$, confirment cette notion, mais l'espèce du Sanglier est nettement plus spécialisée (œsophage non divisé, vulve non très antérieure, cuticule à côtes, groupement de certaines papilles autour du cloaque) qu'O. raillieti, parasite de l'Ane en Afrique.

\section{Bibliographie}

BaIN (O.), 1975. - Redescription de cinq espèces d'Onchocerques. Ann. Parasitol. hum. comp., 50, 763-788.

Bain (O.), Bussiéras (J.) et AmÉGÉE (E.), 1974. - Dualité d'Onchocerca volvulus de l'homme et d'O. sp. Cameron, 1928, du bétail. Nouvelles espèces d'Onchocerques bovines au Togo. C.R. Acad. Sci. Paris, 278, sér. D, 369-372.

Bain (O.), Müller (R.-L.), Khamis (Y.) et Guilhon (J.), 1976. - Onchocerca raillieti n. sp, (Filarioidea) chez l'Ane domestique en Afrique. J. Helm., 50, 287-293.

Bain (O.) et Schulz-Key (H.), 1974. - Les Onchocerques du Cerf européen : redescription d'O. flexuosa (Wedl, 1856) et description d'O. tubingensis n. sp. et d'O. tarsicola n. sp. Tropenmed. Parasit., 25, 437-449.

BaIN (O.) et Schulz-Key (H.), 1976. - Une quatrième espèce d'Onchocerque, O. garmsi n. sp. chez le Cerf européen. Tropenmed. Parasit., 27, 474-478. 
BWangamoI (O.), 1969. - Onchocerca ochengi new species, an intradermal parasite of cattle in East Africa. Bull. epizoot. Dis. Afr., 17, 321-335.

Cleland et Johnston (T.H.), 1911. - Notes on worm nests in australian cattle due to Filaria (Onchocerca) gibsoni and on similar structures in camels. Commonwealth of Australia. Govt. Printer, Reprint 35-58.

Galliard (H.), 1937. - Onchocerca cebei, espèce nouvelle, parasite de buffles du Tonkin. Ann. Parasitol. hum. comp., 15, 430-433.

Gubanov (N. M.), 1964. - Helminthes des Mammifères d'importance économique au Yakut. Moscou: Izdatelstvo «Nauka», 164 p. (en russe).

Johnston (T. H.), 1921. - Onchocerciasis of Queensland cattle. Trans. Roy. Soc. So. Australia, 45, 231-247.

Neumann (L. G)., 1910. - Un nouveau Nématode parasite du Bœuf (Onchocerca gutturosa n. sp.). Rev. Vet., 35, 270-278.

Railliet (A.) et HenRY (A.), 1910. - Les Onchocerques, Nématodes parasites du tissu conjonctif. C.R. Soc. Biol. Paris, 68, 248-251.

Ramachandran (C. P.) et TAN (B. E.), 1967. - Microfilaria of the genus Onchocerca from the skin snips of a domestic Pig in Malaya. Malay. vet. J., 4, 159.

Ramanujachari (G.) et Alwar (V.S.), 1953. - Indian Pig (Sus scrofa domestica) as a host for Onchocerca sp. and Enterobius vermicularis. Indian Vet. J., 29, 329.

SPRATT (D. M.) et Moorhouse (D. E.), 1971. - Onchocerca sweetae sp. nov. (Nematoda : Filarioidea) a parasite of the water buffalo (Bubalus bubalis) from Northern Australia. Zool. Anz., 186, 147-153.

SuPPerer (R.), 1953. - Filariosen der Pferde in Osterreich. Wien. Tier. Marrats., 40, 193220.

Wehr (E. E.) et Dikmans (G.), 1935. - New Nematodes (Filariidae) from North American ruminants. Zool. Anz., 110, 202-208. 